may the therapeutist of to-day declare the three essentials of the treatment of consumption to be 一not microbicide, but-NUTRITION, NUTRITION, and NUTRITION.

EPICYSTIC SURGICAL, FISTULA, FOR THE RELIEF OF VESICAL CATARRH.

Read by Title in the Section of Surgery and Anatomy, at the Fortieth Annual Meeting of the American Medical Association, June, 1880 .

BY JOHN D. S. DAVIS, M.D., OF BIRMINGHAM, ALA.

The treatment of vesical catarrh by suprapubic drainage, irrespective of the causes producing the disease, is not only the most rational, theoretically, but the most available and successful. Such has been the palliative, restorative and curative effect of suprapubic drainage in cases of suppurative cystitis that I have treated by this method, that I have repeatedly resorted to it, with the happiest results.

A close observation of my cases, though at first giving me much anxiety and apprehension, taught me the valued lesson of suprapubic drainage in cystitis. The epicystic fistula gives a desirable continuous drainage, I have not been able to get by any other metbod. The fistula possesses the ability, through the recti muscles, and a possible pseudo-sphincter, to control the urine at all times. The pseudo-sphincter muscle is formed, no doubt, by the fibres of the detrusor urinæ.

I have tried drainage of the bladder by perineal incision, in the treatment of vesical catarrh; but in no instance did I get such results as in the cases treated by a direct opening into the bladder above the pubis.

September, I 888 , I was called by a physician friend, Dr. J. C. Dozier, to see a patient with him. The man, Mr. B., had retention of urine from an impassable stricture of the urethra and enlarged prostate gland. I aspirated his bladder of $2 \mathrm{I} / 2$ pints of alkaline urine, containing pus and mucus. The patient was able to pass a very small stream of urine the next day and would not be operated on for the relief of the stricture. Three months later I was called to see him, and found him suffering from extravasation of urine into the scrotum as the result of an unsuccessful attempt to draw his urine the day previous. The contusion, puncture, etc., resulted in an extensive extravasation into the scrotum, perineum, and above the pubis. I found him with a severe chill, temperature $106^{\circ}$, and pulse 140 . I feared extravasation into the peritoneal cavity and suggested to the family the possibility of having to open the peritoneal cavity at once. I, however, resorted to draining the bladder above the pubis. Seven days after the suprapubic puncture I performed internal urethrotomy for relief of penile stricture, and began the treatment of the enlarged prostate by electrol- ysis. The bladder was irrigated with hot water twice daily for ten days. Electrolysis was used on prostate every third day for ten times, when it was discontinued. The fistula was kept open for thirty days, when it was allowed to close by its own continuity and repair. This was accomplished in about ten days. The man is entirely well and at his business.

Mr. A., æt. 57 years, had been suffering from vesical inflammation for six months. For several weeks before he consulted me he passed his water with much pain. He had rigors and exhaustive night sweats. His temperature would go up for two or three days at a time, and would be followed by two or three consecutive days of only slightly elevated temperature. He had frequent lumbar pains shooting toward the bladder and hip, retraction of right testicle, and tenderness on pressure over the right kidney. Had frequent desire to urinate, with inability to pass his urine. Urethra competent. Prostate gland was slightly enlarged. Urine contained pus and mucus. Average daily amount of urine 39 ozs. After the washing out of the bladder pus would soon accumulate in the viscus, which was conclusive to my mind that pyelitis was causing the progressive emaciation and hectic fever.

Operated for the formation of the direct epicystic fistula for draining bladder and kidney. Bladder was washed out twice daily. Fever subsided on the second day. Patient is entirely relieved from pain; fistula well formed. He returned to his home on the thirtieth day. I hear from him, however, every second day, and he is improving all the time. If the pyelitis had been due to stone in the kidney it would not have disappeared so early. And, too, in such a case the fistula would offer but temporary relief to the bladder only, I am sure he will experience relief so long as he keeps the fistula open and washes out his bladder. By the use of my epicystic fistula $p_{\text {lug }}{ }^{1}$ he will have no trouble in keeping the fistula competent. It is possible that he may in one or two years be able to let the fistula close in safety. It should not be allowed to close until every symptom of the disease has disappeared for many months. Specific gravity of urine June 5, I 889 (forty-nine days after operation), IO2O, no mucus and no pus.

I was captivated by the enthusiastic reports of Dr. Hunter McGuire, ${ }^{2}$ on his coffee-spout fistula for prostatic hypertrophy, and did epicystotomy for the formation of his coffee-spout fistula. Two cases have been reported, ${ }^{3}$ with no better results

'The author's epicystic fistula plug is made to fill the fistula within one-third of an inch of viscus. The plug (which was first suggested to the author by his brother, Dr. W. E. B. Davis), is made of silver or vulcanized rubber and provided with a slight shoulder onefourth of an inch from concavo-convex flange, which is grasped by the skin and held in position. Without the shoulder the recti mus cles throw the plug out and prevent its retention.

2 Virginia Med. Monthly, October, 1888 , pp. $445-456$

3 Virginia Med. Monthly, April, I889, pp. 1-5; Alabama Med. and Surg. Age, April, i889, pp. I92-I98; New York Med. Journal, April
13, I889, pp. 416,417 . 
than have attended the direct suprapubic fistula. I adopted the coffee-spout fistula with the view of practicing it, but I am so thoroughly convinced that it does not possess the advantages attending the direct fistula, that I do not think I will ever resort to it again.

In prostatic hypertrophy, where there is much distension of the bladder, due to retention of urine, and the bladder is prominent above the pubes, a sufficient opening can be made, by a single plunge of a large trocar or knife, for cystoscopic exploration and the formation of the fistula for intra-vesical treatment and drainage. If nothing more than drainage is necessary, the dread and shock of a technical epicystotomy (by a long incision) may be avoided, by giving the patient the benefit of a fistula in the manner suggested. I have resorted to this measure in cases of very old and feeble men, where I could not administer an anæsthetic, with immediate relief to the patient. In conditions of the bladder where, by reason of a long-standing inflammation of the bladder, the viscus becomes contracted, it is necessary to make an incision through the integument, superficial fascia, muscles and transversalis fascia, sufficiently large to allow the bladder to be opened for intra-vesical ocular exploration and operation.

The technique of the operation for the formation of the epicystic fistula, described in my recent paper $^{4}$ before the State Medical Association of A1abama, and published in many of the leading journals of this country, was the result of my enthusiasm over the coffee-spout fistula. My experience with the formation of the direct and coffeespout fistulæ has taught me that the former is always practicable, as it can be quickly made in cases where an anæsthetic cannot be given with safety, and possesses every advantage attributed to the coffee-spout fistula, and is superior to it in the power to retain and expel the urine, by reason of a better power exerted through the recti muscles near the symphysis.

Hence I consider it necessary to retrace my steps for the better consideration of the direct epicystic fistula, which I have formed for exploration, intra-vesical treatment and drainage, and reconsider it under the following heads :

I. Definition of epicystic surgical fistula.

II. Surgical resources in the formation of the epicystic surgical fistula.

I. Preparation for the operation.

2. Anæsthesia.

3. Position.

4. Incision and opening bladder.

4 "Epicystic Surgical Fistula for Cystoscopic Exploration, Intravesical Treatment and Drainage,"' by John D. S. Davis, M.D., April, I 889 . Transactions of the State Med. Assoc. of Alabama, 1889 ; St. I,ouis Med. Review, May 4 and 18, I889; The JoURNAL OF THE AM. MFD. Assoc., May is, I889, pp. 685-703; Ala. Med. and Surg. Age, May, 1889 , pp. 247-256; Virginia Med. Monthly, May, 1889 , pp. I47, I48; Atlanta Med. and Surg. Jour., June, I889, pp. 204-212; The Amer. Prac. and II, I889, pp. 27-29; Cincinnatimes M. Times June, I889, pp. 87,88 .
Y. Med, April, 1889, pp. 21 7-224; N.
5. Intra-vesical exploration and treatment.

6. Toilet and after-treatment.

III. Advantages of the epicystic surgical fistula.

I. Cystoscopic exploration.

2. Intra-vesical treatment.

3. Drainage.

I. Definition of Epicystic Surgical Fistula.Epicystic surgical fistula is the title here given to a suprapubic fistula into the bladder, created by the surgeon for exploration, intra-vesical treatment and drainage. A fistula which, acting as an artificial urethra, is capable of giving free access to the inside of the bladder for cystoscopic exploration, to provide a ready, convenient and comfortable means of emptying the bladder at will; gives the surgeon a competent opening into the viscus for exploration of ureters; intra-vesical applications; drainage in pyelitis and in pyelonephritis.

It constitutes an essential element in the speedy and complete evacuation of the contents of the bladder in all epicystic operations, and imitates nature in the restoratton of its own continuity and repair as the pathological changes within the bladder subside.

II. Surgical resources in the formation of the epicystic surgical fistula.

I. Preparation for the Operation.-All hair is to be shaved from the pubes, and all the details of antiseptic surgery are to be carried out so far as cleaning the pubes and abdomen.

Except in those cases of hypertrophy of the prostate gland; paresis and atony; neurosis of the bladder, and in all forms of bladder inflammation accompanied with distension of the bladder into prominence above the symphysis, where immediate epicystotomy, by puncture with a trocar or knife, is necessary, two assistants, though not necessary, may be of valuable aid.

A temperature of $80^{\circ}$ or $85^{\circ} \mathrm{F}$. should be maintained in the operating-room from the beginning to the end of the operation. The bladder is emptied and thoroughly washed with warm water. When the water returns clean, the bladder is slowly distended with warm sterilized water, thrown in by means of a fountain syringe, with nozzle in urethra-with a degree of pressure sufficient to distend the bladder to its utmost capacity-which can never be too great for the resistance of the bladder. It is better to fail in filling the bladder than to distend the bladder beyond the limit of competency. Indeed, it is not necessary to fill the bladder to any degree of resistance. I have operated when the bladder was in an irritable condition and would not tolerate distension greater than the capacity of 2 ozs., and had no difficulty in avoiding the pre-vesical fold of peritoneum or finding the bladder. The water is secured in the bladder by tying the penis at the base with a rubber tube. 
A colpeurynter is next to be well oiled and inserted into the rectum-the rectum having been previously emptied by enema-and filled with warm water. This distension brings the bladder into view above the pubis.

2. Anesthesia.-My preference for chloroform is the result of personal experience with it. It is not free from objection, as its depressing effect on the heart is well known. The operation usually occupies from five to ten minutes; and hence its prolonged use would be unnecessary and uncalled for. The objections to ether are the suppression of the excretions, and the frequency with which bronchitis is produced when administered to persons advanced in years. The best course to purste, when the operation is prolonged, is to follow the use of chloroform by ether.

In old men where there is much distension from retention of urine, due to hypertrophy of prostate, to avoid the dangers of an anæsthetic, I make the fistula by one plunge with a large trocar or with a knife, the shock never being noticeable.

If the operation requires the administration of an anæsthetic, the patient must be kept profoundly under its influence from the first incision until the superficial wound is closed.

Position.-The patient is placed on the back on an ordinary operating table with the legs extended, as if in a position of perfect comfort and rest. Many surgeons claim advantages in the position recommended by Trendelenburg. Eigenbrodt emphasizes the fact ${ }^{-5}$ that the elevation of the pelvis in Trendelenburg's position ${ }^{\circ}$ helps the surgeon to avoid the prevesical peritoneal fold at the time of the incision of the bladder.

It possesses no advantage over the ordinary flat-back position. With two openings in the bladder for a continuous stream of clear water, every part of the bladder may be illuminated with the electric surgical light, and thus the surgeon is enabled to examine the entire intra-vesical wall. Undoubtedly the position recommended by Trendelenburg possesses advantages which to the author, more than to myself, make it highly ideal. As for myself, I prefer and recommend the flatback position.

4. Incision and opening Bladder.-I have three methods for making the incision, viz.:

$a$. When the distension is great and no intravesical operation necessary, the opening is made with a trocar, withdrawing the stylet and replacing it with a rubber catheter, after the introduction of which the canula is withdrawn, leaving the catheter in the bladder. It is necessary, sometimes, to enlarge the external or cutaneous opening to prevent a closure of the catheter by compression and to render the external opening sufficiently large.

L. c., p. 72. Cf. I,ang, Med. News, December 4, 1886

${ }^{6}$ In Trendelenburg's position the patient's legs are held over the shoulders of an assistant with the body resting on an incliue table, much in the position in which hogs are hung for spaying. $b$. The bladder may be opened, when distended, by a direct incision with the knife, in the median line, with cutting edge towards the symphysis pubes. The knife is withdrawn and a catheter is introduced, through the wound, into the bladder.

c. A perpendicular incision, I or 2 inches, is made in the median line above the symphysis pubes. The recti muscles are separated to symphysis. If the pyramidalis muscles are in the way, the fibres should be cut. The transversalis fascia is divided on a grooved director from symphysis to upper margin of superficial wound. Instead of following Guyon's manœuvre, I catch the bladder with a tenaculum on a line with the symphysis, through the prevesical fat, and cut through with a bladder knife into the bladder with one smooth, clean incision, to prevent undue disturbance of the cellulo-adipose tissue between the bladder and pubes, and avoid infiltration. I have never seen a case where it was necessary to put up the prevesical fat, and with it the peritoneal cul-de-sac. If the bladder is caught on a line with the symphysis and cut downwards, no fears need be had for the peritoneum. Cutting this prevesical fat prevents its after dropping down over the opening into the bladder, and acting as a valve to prevent easy escape of urine and causing infiltration. And, too, such a procedure gives a smooth incision throughout, and it is almost impossible to have infiltration, even when no drainage tube is left in the bladder, and the urine is left to flow out through the fistulous track and taken up by a layer of absorbent cotton. In making the incision into the bladder, little attention is to be paid to any vein or veins which are sometimes met with. If cut, they will stop bleeding when the bladder is dropped back and the rectal bag removed. The operation is usually bloodless in the sense of hæmorrhage. I have operated without the patient losing more than I drachm of blood.

5. Intra-vesical Exploration and Treatment.With the provisions of the puncture or incision the bladder can be examined with the cystoscope and surgeon's light. If tumors be found, if practicable they should be removed; villous growths and any foreign body found should be removed. If nothing is found in the bladder, the fistula, in the absence of malignancy, will be all that is required to remove the cystitis.

Under the provision of the last incision, the finger is carried into the bladder and a thorough search made for any tumors, villous growths, or foreign bodies which should be removed. The rubber around the penis is now untied and the bladder emptied and washed out with hot sterilized water.

6. Toilette and After Treatment.-Under the provisions of the last method of making the incision, the bladder is now allowed to drop back into the pelvis, and the superficial wound so 
closed by two or more sutures (including the skin and superficial fascia only), in the upper portion of the incision, as to leave a fistulous track of equal size from the bladder to lower portion of the superficial incision.

A large rubber catheter is now to be introduced into the bladder through the opening, and its distal extremity allowed to enter a urinal placed in the bed between the patient's thighs, or preferably at the patient's side. Prof. F. Trendelenburg, director of the surgical clinic of the University of Bonn, proposed, for draining the bladder in supra-pubic lithotomy, the $T$ tube in latero-abdominal position and open wound treatment as the simplest, safest and best. He makes an antiseptic dressing of iodoform gauze around the $T$ tube. There can be no real necessity for a tube of any kind to be introduced into the bladder for the purpose of conveying the urine from the bladder to prevent infiltration, except in the puncture operation, when it is necessary to let the catheter or canula remain in for a period of six or eight hours, for the formation of the fistula. The smallness of the fistula might be a cause for extravasation, though quite improbable. To prevent irritation of the skin, the urine is kept acid by the administration of citric acid or some other more palatable acid drink. No better antiseptic than the acid urine can be secured for the constant bath of the parts. It should be allowed to flow out through the wound and absorbed by a pad of absorbent cotton placed loosely over the wound, and removed as often as soiled by the outflowing urine. By this method of emptying the bladder, no possible small amount of urine can be impeded in its outflow, which is the case around and outside of the tube when catheter or tube is left in for any length of time-a source of no little annoyance at times. This little collected or retained urine, around the outside of the tube alone, I have seen produce a hard chill and elevation of temperature, and becone for the time an immediate, alarming and aggravative source of trouble. I never have seen the skin made sore or chafed by the outflowing urine in epicystotomy, or from its after escape through the surgical fistula.

The bladder should be washed out twice daily with hot sterilized water, by means of a fountain syringe, with its nozzle introduced into the urethra, the water escaping through the epicystic fistula and guided into a bed pan under the patient.

The superficial stitches are taken out at the end of one week, and intermittent catheterization by the fistula is then resorted to, for the sole purpose of training the fistula and to prevent its rapid closure, It is not necessary to catheterize for the purpose solely of drawing off the urine. In some cases I never drew the urine save for the purpose of analysis, but occasionally introduced a rubber bougie or fistula plug to prevent the closure of the fistula. The drainage by the fistula alone is admirable, and the fistula will be well formed in twenty or thirty days, competent to retain urine without dripping and to allow its escape in a good projecting stream at will. With no tearing of the tissues, and with a clean cut, the drainage is perfect and the dangers are nil.

III. Advantages of the epicystic surgical fistula.

I. Cystoscopic Exploration.-One of the great difficulties in the cystoscopic exploration of the bladder is the presence of pus, mucus and sometimes blood, which renders it exceedingly difficult to maintain a translucency of the fluid used to distend the bladder. By means of a simple fountain syringe a constant current of clear water may be kept within the bladder, so essential to a complete observation of the trigonum Lieutaudii, the most interesting part of the viscus, the ureters, and to examine any affection of that viscus. The fistula may be made for temporary purposes of cystoscopy. Diagnostic purposes are met by the possibility of immediate detection of all local conditions, such as tumors, calculi, foreign bodies, neoplasms, the collection of fluids from the ureters, etc.

2. Intra-vesical Treatment.-Having, by means. of the epicystic exploration, revealed the true nature of the intra-vesical trouble, the treatment resolves itself into the immediate necessities of the case. For instance, prostatectomy may be necessary; villous papilloma may be found, and should be remedied; pedunculated growths may be found, which should be removed by the scissors, or Paquelin's cautery, etc. In such cases the opening in the bladder sufficient to introduce a finger, should be enlarged downwards under the symphysis pubes, and the operation indicated should at once be performed. The object of the formation of the permanent surgical fistula is to meet the after indications in such operations, the details of which do not properly come within the province of this discussion. However, it is sufficient to state, what is reasonable and practicable, that a better means by which the intravesical wall can be reached and treated therapeutically has not yet been devised.

3. Drainage.-Permanent after-drainage in all intra-vesical operations cannot be necessary, but it is highly essential to secure good and sufficient drainage until the paravascular tissue is disengorged, the cystitis is relieved and the urine becomes normal and passes per urethra unobstructed. And until this end is obtained, complete artificial arrangement for the escape of the contents of the viscus must be made. In such cases of prostatic hypertrophy or malignant growths, when removal of the obstruction is impossible or contraindicated, the epicystic surgical fistula is clearly indicated and essentially neces- 
sary. It meets every possible indication for their way, on the last day, his wife and oldest local treatment and gives the only controllable daughter were taken sick, and on their arrival ready and free drainage to viscus and kidneys. that evening $I$ was consulted and found them Urinary back pressure, as the result of incompe- both very ill of scarlatina anginosa. They had tency of the urethra from the various immovable moved their family and household goods into a prostatic troubles, is often an immediate and house with a family residing in that town. A remote cause of surgical kidney, which can only few days later his other and youngest daughter be removed or relieved by supra-pubic drainage. was attacked with the same form of disease, and

It is also indicated in conditions of the bladder a few days later three children of the family where of long standing cystitis, where capillary stasis they were staying were also attacked, two with attending the inflammatory process results in the same form of scarlatina, and the third case paresis. When the urethra is competent, the was very severely seized with scarlatina maligna. supra-pubic fistula affords the best means of $I$ at once ordered all clothing and household goods washing out the bladder in chronic cystitis.

The operation has been performed when the urethra would admit a number 30 (A) sound, simply for the purpose of washing the bladder without the necessity of introducing a catheter twice a day, which causes great irritation in these cases. As stated, with the fistula it is only necessary to introduce the nozzle of a fountain syringe into the urethra, when the water will pass into the bladder and out at the fistula, without irritation to the prostate and bladder, and always with great relief to the patient. The bladder can be washed for bours at a time, when the patient is suffering, with complete relief from all pain.

\section{SCARLET FEVER.}

Read in the Section of Diseases of Children at the Fortieth Annual Meeting of the American Medical Association, June, 1889 .

BY C. R. EARLEY, M.D.,

OF RIDGEWAY, PA.

removed to a large farm barn and placed on a

scaffold over the threshing floor, consisting of

round poles which admitted of free airing, and

here every means was used to disinfect all goods

and clothing. The house where they were staying was also disinfected, and the patients freely bathed and quicklime placed plentifully in every room. All the cases at once began to improve and were soon well, and not another case was produced in that place. There was no case in the old home from which they had come, before or after their removal. I of course placed this series of cases side by side with the reports of Dr. Rush and others on like cases of yellow fever.

The disease was produced in that family by the condition of the clothing and household goods, that had been stored away in an old, damp and unused room in the house they moved from, where they were shut up from air or light and were filled with mould and dust, and the whole mass of mould, dampness and other filth was packed into boxes and opened upon their arrival in Pennsylvania. Thus those that took them from the boxes

A genus of disease in the class Pyrexia, and order Exanthemata of Cullen. It is an eruptive fever particularly affecting the skin and mucous membranes of the mouth and fauces, and by a majority of writers on the subject is considered as a contagious disease. This I look upon as very doubtful, or at least it has not proved the fact to me in my practice for the last forty-four years. I have known one or more of the same family to have well marked cases of the disease, while others were entirely exempt, and while some families in the locality were every day exposed to it and escaped an attack, other families that prevented their members from going amongst it, when the disease prevailed, or having any communication with those that were sick of scarlet fever, were subject to the disease in all its forms.

The only case I ever saw in which contagion was suggested to my mind was that of a family of four, a man, his wife, and two daughters, residing on a farm about eighty miles north of our town. On the I $3^{\text {th }}$ day of January, 1869, he packed his household goods in a two-horse sled and moved for Earley, Elk County, Pa., arriving there on the evening of January I4, 1869. On in the room at the old home and packed them in the boxes were taken sick with scarlet fever, and all that assisted or looked over the unpacking of the same on their arrival in Elk County, Pa., were also seized with the same disease, and after all these goods were removed to the barn and thoroughly disinfected, not another case existed or was produced, and those that were sick at once recovered; thus showing the cause to be from the condition of the goods unpacked and brought with them and again unpacked without cleansing freely, airing and disinfecting.

In my entire practice I have never known any outbreak of scarlet fever to be produced by any other than local causes. In the country, where the epidemic breaks out in localities where there are no communications with the outside country, where no person has been from home and returned, and no one from other towns and localities has visited, we have in such cases, in my opinion, positive proof that the disease is produced by local causes, especially when, as has always been the case in my practice in the country and small towns and among farmers, lumber camps, mining towns, etc.; at elevations from 750 to $2,35^{\circ}$ feet above 